\title{
Bu open Improving ethnic monitoring for telephone-based healthcare: a conversation analytic study
}

\author{
Geraldine M Leydon, ${ }^{1}$ Katie Ekberg, ${ }^{1}$ Moira Kelly, ${ }^{2}$ Paul Drew ${ }^{3}$
}

To cite: Leydon GM, Ekberg K, Kelly M, et al. Improving ethnic monitoring for telephone-based healthcare: a conversation analytic study. BMJ Open 2013;3:e002676. doi:10.1136/bmjopen-2013002676

- Prepublication history and additional material for this paper is available online. To view these files please visit the journal online (http://dx.doi.org/10.1136/ bmjopen-2013-002676).

Received 2 February 2013

Revised 8 April 2013

Accepted 19 April 2013

This final article is available for use under the terms of the Creative Commons Attribution Non-Commercial 2.0 Licence; see http://bmjopen.bmj.com

\footnotetext{
${ }^{1}$ Primary Medical Care and Population Sciences, University of Southampton, Southampton, UK

${ }^{2}$ Institute for Health Sciences Education, Queen Mary University of London, London, UK

${ }^{3}$ Department of Social Science, Loughborough University, Loughborough, UK
}

Correspondence to Dr Geraldine M Leydon; G.M.Leydon@soton.ac.uk

\begin{abstract}
Objectives: Medical and healthcare organisationsincluding the national cancer support and helpline organisation that is the subject of this study-are expected to collect and monitor information about the ethnicity of their client populations. Information about ethnicity is important for a variety of reasons, including monitoring need and targeting healthcare services appropriately. Previous survey and interview research has suggested that collecting ethnicity data from service users can be incomplete and of variable quality -pointing to a need for an improved understanding of the (interactional) difficulties involved when call-handlers ask callers about their ethnicity.

Design: This study analyses a corpus of real-life audio-recorded calls to a national cancer helpline in the UK, focusing on the way that call-handlers collect the ethnic monitoring data.

Setting: A major national cancer helpline in the UK. Participants: A sample of 273 recorded calls were recorded, of which 267 were frontline calls in which call-handlers are expected to ask the ethnicity monitoring question.
\end{abstract}

Results: Findings suggest that caller uncertainty about how to answer the question, resistance to answering and call-handler presumption can compromise the effectiveness of ethnic monitoring. It is likely to be improved by changing how the ethnicity monitoring question is asked. Changes include avoiding open question formats to ease caller uncertainty; offering callers a rationale (account) for the question to minimise resistance and confirming the accuracy of the ethnic category recorded.

Conclusions: We recommend that telephone-based healthcare personnel avoid asking the ethnicity monitoring question in an 'open' format; instead, a question containing a (short) standardised list can assist callers in responding. A training tool has been developed that applies this and other findings, with a view to improving ethnic monitoring.

\section{INTRODUCTION}

Medical and healthcare organisationsincluding the national cancer support and helpline organisation that is the subject of this study-are expected to collect and

\section{ARTICLE SUMMARY}

Article focus

- Organisations are mandated to collect ethnicity data from their users, including in telemedicine; however, collecting ethnicity data from service users over the telephone can be problematic.

- Most previous research has examined surveybased collection of ethnic monitoring data.

- This study analyses a corpus of real-life audiorecorded calls to a national cancer helpline in the UK, focusing on the way that call-handlers collect the ethnic monitoring data.

\section{Key messages}

- Caller uncertainty about how to answer the question, resistance to answering and call-handler presumption can compromise the effectiveness of ethnic monitoring.

- Recommendations to improve the consistency and validity of telephone-based ethnic monitoring include: avoiding open question formats to ease caller uncertainty; offering callers a rationale for the question to minimise resistance and confirming the accuracy of the ethnic category recorded.

- On the basis of the study findings, we provide a novel online training workshop for telephonebased ethnic monitoring.

Strengths and limitations of this study

- Findings based on direct observation and analysis of real-life recorded cancer helpline calls.

- Recommendations about effective practice are derived from actual practice, and are not theory driven.

- Analysis based on one cancer helpline, although our findings resonate with research on ethnic monitoring in a range of environments, which points to the likely conceptual transferability of findings.

monitor information about the ethnicity of their client population. Information about ethnicity is important for two principal reasons. First, healthcare organisations have an interest, indeed a duty, to ensure equality of access to all healthcare services (ie, subsumed by the duty of all public bodies to 
consider the equality implications of the policies that shape their work $^{1}$ ). Second, information about the ethnicity of patient populations can "provide vital information about the patterns of health and social indicators and provide an essential foundation for tackling health inequalities between different populations." ${ }^{2}$ Furthermore, information about the ethnicity of individual patients/callers can enable healthcare organisations to target services, guidance and support appropriately, according to the known epidemiological patterns of incidence of particular diseases. ${ }^{3}{ }^{4}$ However, there is evidence across a range of healthcare organisations that frontline staff experience difficulties in eliciting this information, ${ }^{5-8}$ and that partly as a consequence of these difficulties the information collected about ethnicity can be incomplete, inaccurate or unreliable. $^{9}$ Nevertheless, despite the difficulties associated with categorising ethnic identity, ${ }^{10-12}$ and those associated with collecting accurate information from patients/callers, the clear recommendation is that "only by using the data (of self-assigned ethnicity), even when of suboptimal quality, and remaining close to it can healthcare organizations drive up quality." 13

There is widespread recognition that little is known about how frontline staff actually collect information about ethnicity-how they ask the relevant question(s), how patients/callers respond and how difficulties are managed. While it is known, for instance, that ethnic self-identification using open questions can be confusing for participants and can lead to errors in responses, ${ }^{781415}$ nevertheless there is an acknowledged gap in our understanding of the process through which information about ethnicity is actually elicited and gathered. ${ }^{15} 16$ The literature clearly points to a need for more informed guidance on effective ethnic monitoring, based on detailed qualitative observational information about how the ethnicity question is asked and what difficulties arise. However, the majority of previous research has focused on survey-based ethnic monitoring, rather than the telephone-based questions that feature increasingly in tele-health. There appears to be no consistent way in which call-handlers working in the National Health Service (NHS) or other healthcare environments are trained to ask ethnicity questions over the telephone. Indeed, the majority of research has not been observational, and thus not capable of investigating precisely how the ethnic monitoring question is asked and detailing exactly how problems arise and, importantly, how they can be avoided or resolved.

\section{ETHNIC MONITORING ON A CANCER HELPLINE}

This is part of a larger study of a major national cancer care service, focusing on their helpline operation. This organisation acknowledged difficulties in eliciting information about the ethnicity of callers, as evidenced for instance in their observation that in 2011 the ethnic monitoring question was not asked in $21.7 \%$ of calls. In addition, informal discussion with helpline staff indicated that asking the ethnicity question was often experienced as difficult. All helpline calls are initially fielded by 'frontline' call-handlers who collect routine data, including the caller's name, date of birth, address, marital status, contact details and ethnicity.

Although this cancer helpline organisation adopted the recommended Census ethnic categories as devised by the Office for National Statistics (shown in online supplementary appendix 1), frontline call takers typically asked about callers' ethnicity using these broad groups: White, Mixed, Asian, BlackAfrican/Caribbean and other. Call-handlers are not guided in how to ask the ethnicity monitoring question.

\section{OBJECTIVES}

Through detailed analysis of audio recordings of naturally occurring and routine telephone interactions to this national cancer care helpline, we aimed to:

1. Identify the different ways in which ethnic monitoring questions were asked by helpline staff over the telephone.

2. Identify the difficulties that are experienced and presented in asking and answering this question.

3. Identify from call takers' current practice those techniques that are effective in avoiding or overcoming those difficulties to improve telephone-based ethnic monitoring.

\section{METHOD}

The data for this study were collected between July 2010 and August 2011. A sample of 273 recorded calls were recorded, of which 267 were frontline calls in which callhandlers are expected to ask the ethnicity monitoring question; these frontline calls average $10.5 \mathrm{~min}$ (these calls were often then triaged on to specialist nurses. ${ }^{17}$ Written subject consent was obtained from callers and callhandlers (see online supplementary appendix 2 for recruitment). Calls were transcribed in detail using standard conversation analytic transcription conventions that capture the timing and delivery of talk (when and how things were said ${ }^{18}$; transcript notations are shown in online supplementary appendix 3). The audio-recorded calls and transcripts were analysed systematically to identify key activities in interaction and to diagnose where interaction seemed to falter; and to identify techniques that may pre-empt or remedy such difficulty ((for a review see refs. 19-23)). The Faculty of Medicine at the University of Southampton reviewed the study and awarded Ethics and Governance approvals (reference: SOMSEC060.10).

\section{RESULTS}

In the following analysis, we identify problems in the telephone interactions when asking the ethnicity question, including caller uncertainty about how to respond, in particular how to self-identify and what constitutes an 
ethnic category, caller resistance to providing an answer to the question and, finally, call-handler presumptions about caller responses and their impact on validity. We consider each of these in turn and propose a particular question formulation to improve consistency, validity and the smooth progression of calls.

\section{Caller uncertainty and the open question format}

While frontline call-handlers had available the menu of approved ethnic categories listed in online supplementary appendix 1, they tended to ask an open question designed to elicit callers' ethnic self-identification. Use of the open question format often leads to caller uncertainty in terms of how best to answer the question. The difficulties callers have in answering the question reveal some misunderstanding about the meaning of 'ethnicity' and what counts as an appropriate 'ethnic' category. Fragment 1 provides an initial illustration of some of the trouble that can be generated by an open question formulation when collecting ethnicity data. All the data fragments presented are representative of the recurrent patterns found in the corpus of calls analysed.

(1) [102-712-492 open Q]

1 ISO: Oh\#ka::y (2.0) er $\uparrow$ and how do you describe your ethnicity?

$2(0.7)$

3 ISO: Your [eth]nic background?

$4 \mathrm{C}: \quad$ [um ]

$5(0.2)$

6 C: Um I'm Scot- well $>$ Scottish $<=$ British.

In Fragment 1, the call-handler poses the open question "how do you describe your ethnicity?" There is some evidence that the question is being treated as somewhat delicate by the call-handler with the pause of $2 \mathrm{~s}$ before they formulate the question (in conversation, a gap of more than 1.0 is unusual and tends to indicate some form of interactional 'trouble'). The stumbling production also suggests that the question is problematic for the callhandler and further marks the question as 'delicate'. The caller clearly has difficulty in answering the question immediately (line 2, 0.7 gap) and the call-handler, clearly aware of the caller's difficulty, repeats the question (line $3)$. However, even the re-elaboration is inadequate to solicit an immediate response from the caller (line 5, 0.2 gap). Finally, at line 6 , the caller produces a response with 'um I'm Scot- well $>$ Scottish $<=$ British' (trouble-free interaction would ordinarily entail smooth transition between speakers with no notable intra turn silence). The perturbation in the caller's turn suggests a struggle to respond and they provide nationality rather than ethnicity.

Fragment 2 provides further evidence of the difficulty often encountered when the ethnic monitoring question is formulated in open format.

(2) [102-748-150 open Q]

1 ISO: Um $\uparrow$ and how would you describe your ethnicity::?

2 (.)

3 ISO: Or your ethnic origin.
4 C: Er ju- just uh (0.5) British=English whatever $\uparrow I$ don't [know.]

5 ISO: $\left[{ }^{\circ} \mathrm{Okay}^{\circ}{ }^{\circ}{ }^{\circ}\right.$ thank you ${ }^{\circ}$.

The call-handler uses an open formulation which invites a response from the caller in their own words 'Um and how would you describe your ethnicity?' (line 1). Again, the question start is produced with slight hesitation ('um'), and there is again a brief gap in interaction (line 2), and as in Fragment 1 the call-handler reformulates the question 'or your ethnic origin' (line 3). The caller provides a response (line 4), but as before they offer a 'nationality' category rather than 'ethnicity'. In addition, the caller displays some difficulty in formulating their answer with stumbling production ('Er ju- uh') and a pause (0.5). The uncertainty experienced by callers when faced with an open format ethnicity monitoring question is clear.

Fragment 3 provides a penultimate example of how callers can display uncertainty over the conceptualisation of ethnicity, when asked in an open format.

(3) [102-810-886 open Q]

1 ISO: and $\uparrow$ how would you describe your ethnic origin

2 C: I'm (.) I'm church of England I'm the verger at the church here

3 ISO: okay and would you be $\uparrow$ white (.) $\uparrow$ mixed (.) †asia:n (.) asian british,

$4 \mathrm{C}$ : white huh huh

The call-handler asks the ethnic monitoring question in an open format, this time using the formulation of 'ethnic origin' (line 1). The caller is clearly hesitant at first (cutting off after her initial "I'm" and pausing before continuing her turn) and in the remainder of her turn, rather than provide her ethnic origin, the caller provides her religion 'Church of England' and position of church 'verger'. The call-handler must then reask the question in order to solicit a more appropriate response and elects to use a survey list of response items for the caller (lines 4-5). The caller is now in a position to provide an appropriate response ('white'), which is notably followed by laughter (line 6); it is likely that the laughter orients to her prior response which revealed a clear misunderstanding of the initial question. ${ }^{24}$ The ethnicity question, when asked in an open format, can lead to caller uncertainty about how best to respond and linked to such uncertainty can be embarrassment and/ or delicacy for both the call-handler and the caller.

Fragment 4 offers one final example of the disruption that can flow from an open format and demonstrates how reverting to a survey list of options can side-step the problem of uncertainty and successfully elicit the type of response required.

(4) [102-796-220 open Q]

1 ISO: Two four fifty three:, and your ethn $\uparrow$ icity? =your ethnic background,

$2(0.2)$

3 C: Pardon?

$4(0.2)$ 
5 ISO: Your ethnicity.

6 (1.0)

7 C: Wha- [wha-]

8 ISO: [Er ] wh- whi:te mixed [Asian]

$9 \mathrm{C:} \quad$ [Oh I'm] whi:te.

10 ISO: You're white $\uparrow$ okay and can I take a telephone number from you?

The call-handler asks the question in open format "and your ethnicity=your ethnic background (lines 1-2). It is noteworthy that after "your ethnicity", the callhandler rushes through into an alternative question formulation (symbolised by the '=' sign), "ethnic background". That they do this without giving the caller an opportunity to respond to the first formulation again provides evidence that the call-handler has difficulty with how best to ask the question and is treating it as a delicate interactional task. As with the previous three examples, there is a gap in talk where the caller could have provided a response, and this absence provides a signal that the question might again be problematic for the caller. Indeed, the caller provides an explicit request for the clarification 'pardon' (line 4) and following another brief gap, the call-handler responds to the caller's request by repeating the question 'your ethnicity'. The caller still displays some trouble providing an answer 'wha- wha' and, finally, the call-handler shifts tack to move from an open question format to offer a list of options (line 9). The caller's 'oh' indicates a realisation of what is required, further evidenced in their eventual concise response 'I'm white'.

\section{Caller resistance to the ethnic monitoring question}

Next, we provide an example of how callers may resist the ethnic monitoring question. For example, callers may struggle to determine the relevance of the question for them, which in turn can lead to disruption to the telephone interaction.

(5) [102-678-305 account]

1 ISO: .Hhhh and can I ask your ethnic backgrou:nd as in are you whi:te mixed bla:ck

$2(0.6)$

3 C: Er one question that I take exception to: um because it's of no:: r:elevance believe

$4>$ you me $<$ when. hhhh I've needed abroad lifesaving o:pe:rations I've never a:sked .hh

5 what the ethnic background of the blood that they gave me is two questions is is it my

6 ty:pe and is it clean so

7 ISO: Yeah

$8 \mathrm{C}$ : Um so tha- that is a comple::te to my mind a n: onsense

In Fragment 5, the call-handler asks 'can I ask your ethnic background as in are you white mixed black' (lines 1-2). On this occasion, the call-handler does make it easier for the caller by providing a survey list of options, but this particular caller displays resistance to the question and across lines 4-10 they provide a lengthy explanation for deferring their response.
Analysis of the corpus and even of this particular call provides a solution to such caller resistance. Fragment 6 follows on from the caller resistance in 5 above.

(6) [102-678-305 account]

11 ISO: Okay that's fine if you don't want to answer I just tell you why we ask it it's

12 just so [organization name]can see who we're helping and reaching .hhh see if there's er

13 identify any gaps in the communities where

14 [we (could do) more work ]

$15 \mathrm{C}$ : [O:kay what are the (.) the:] options a- a:gain

When an account is offered by a call-handler as to why the ethnic monitoring question is being asked, a caller is less likely to resist responding. In this particular example, an account prior to the ethnic monitoring question occasions a turnaround from the caller, providing prima facie evidence that providing a preaccount for the question can help to reassure callers about the merits of the question, minimise resistance and, in turn, solicit a response.

Finally, Fragment 7 illustrates the practice of providing a preaccount, which very neatly avoids caller resistance. In addition, use of a survey list of options side-steps the problem of caller uncertainty. This case clearly illustrates the potential for a smooth progression of the call when these elements are incorporated.

(7) [102-601-683 recommended]

1 ISO: And just so $>$ [organization name] can $<$ see what areas of the community we're

2 reach:ing, hh how would you define you:r ethnicity: are you white mixed Asian or asian

3 Brit [ish]

$4 \mathrm{C:}$ [um] white British white

5 ISO: .hhh (0.8) okay and where did you find ou:r number today

6 C: .hh On the website

In Fragment 8, the call-handler formulates the ethnic monitoring question in three parts (lines 1-3). First, a preaccount is provided 'just so [organization name] can see what areas of the community we're reaching' (lines 1-2); this is then followed by the question 'how would you define your ethnicity' (lines 2-3), and a third question part provides a survey list of options for the caller to choose from 'are you white, mixed, Asian, or Asian British' (line 3). The caller responds in overlap with the answer 'white British white' (lines 4-5). Unlike previous examples, there is no resistance or uncertainty, as evidenced by the smooth progression of the call with no hesitation, no stumbling production and no delay in providing a response. Moreover, it is likely that the caller's ethnicity was correctly recorded by the call-handler.

\section{Presuming ethnicity}

Some call-handlers' questioning can contain presumption and this can also have consequences for the quality of the ethnic monitoring process.

(8) [102-744-793 presumed]

1 ISO: >And how< would you describe your ethnic background: is that white?

$2 \mathrm{C}:$ Yeah. 
3 ISO: ${ }^{\circ}$ background yeah ${ }^{\circ}$

In Fragment 8, the call-handler asks the monitoring question in such a way that presumes a 'white' response; 'And how would you describe your background: is that white?' (line 1-2). While the call-handler begins to formulate the question in open format, they then tag the candidate response of 'white' to their question. This formulation interactionally prefers a 'yes' response from the caller (anything other than this would involve disconfirmation and potential disaffiliation) and the question does indeed elicit a socially preferred 'yeah' response (line 3).

In Fragment 9 (seen earlier in Fragment 1), we can see another case of presumption in the call-handler's response to the caller's answer to the question.

(9) [102-712-492 open Q]

C: Um I'm Scot- well $>$ Scottish $<=$ British.

ISO: Whi- white British $\downarrow$ okay .hhh e::r c $\uparrow$ an I take a telephone number?

The call-handler recategorises 'nationality' into ethnicity ('Whi-white British') (line 7) and in so doing they presume a 'white' response.

Presumption can negatively impact the data collection process. Not checking the accuracy of the category recorded before proceeding to other activities can further compromise the data gathered.

(10) [102-600-944 accuracy]

1 ISO: .hh A:nd this is a demographic question for us Acacia, .hh now how would you

2 describbe your ethnicity white? Mixed? Asian Asian British? .hh black or [bla]
3 C:
and ha:lf
4 Arab.
5 ISO: So I would say Asia:n yea:h
$6 \mathrm{C}: \mathrm{Mm}$.

In Fragment 10, the call-handler states 'and this is a demographic question for us, how would you describe your ethnicity white, mixed, Asian, Asian British, black or' (lines 1-3). Thus, unlike the previous data fragments, the call-handler first briefly tells the caller what type of question is about to be asked, and they produce a survey list of options for the caller, which helps the caller produce a response without difficulty. Indeed, in overlap, the caller states 'I'm half (.) I'm half English and half Arab' (lines 4-5). However, the call-handler interprets the response by stating 'so I would say Asian' (line 6) to which the caller responds with a minimal confirmation 'Mm' (line 7). The information provided suggests that this category is incorrect and checking the accuracy of their interpreted category may have elicited a reformulation from the caller and optimised the validity of the monitoring (in the 2011 census in the UK, 'Arab' was listed as an ethnic group under the 'Other' category.).

\section{DISCUSSION}

'Information is the bedrock of a good health service. Improving health and reducing inequalities require information to support needs assessment, which should then be reflected in the planning and commissioning of services'. ${ }^{3}$ We aimed to identify aspects of cancer helpline telephone interactions that may be disruptive to the task of collecting good quality ethnic monitoring data. Analysis suggests that when call-handlers ask the ethnicity question in an 'open' format, callers display uncertainty (hesitate, pause, etc) about how best to respond. These difficulties in responding can be avoided by asking the question instead as a survey-style question with a list of standardised options (quite which options are used will in part be driven by individual organisations).

Asking about ethnicity can be a delicate activity and call-handlers evidently have concerns about how best to ask the question without causing offence, and callers may have difficulties understanding why such a question is being asked. Providing a preaccount explaining why this information is being collected can make it easier for call-handlers, minimise the potential to offend service users and head off caller resistance. A preaccount clearly signals to callers why the question is being asked and how the information gathered will help.

Questions that contained presuppositions about ethnicity-often presuming 'white'-featured in the corpus and it is clear that this can lead to the collection of inaccurate ethnicity demographics. The caller might just produce the preferred response of "yes" even when, for example, they are of a 'mixed' ethnicity. This is consequential for two reasons. First, the proportion of callers from a 'mixed' background will be under-reported. Second, presumptive questions have the potential to cause offence if the caller's answer is in fact "no". Indeed, this could prove to be uncomfortable for both the caller and call-handler.

\section{How this relates to previous research and other helpline environments}

Our findings provide authentic real-life observational examples that support previous research on ethnic monitoring. ${ }^{14} 15$ The findings also support previous survey and interview research, ${ }^{4} 7811$ suggesting that respondents can find ethnic self-identification using an open response format difficult and confusing. These findings suggest that respondents require clear guidance and a choice of ethnic categories in order to produce more stable responses to the ethnic monitoring question. In contrast, it has been suggested that open-format questions may be better at capturing 'super-diversity'.25 However, while this may work well in a survey context, our findings suggest that it may not be appropriate for healthcare telephone environments. Our study has demonstrated that open questions can compromise the quality of data with regard to ethnicity, in part because call-handlers were evidently uncomfortable asking open questions about ethnicity, and because callers appeared to display some uncertainty about how best to respond. 
Translating the research into practice

Training in ethnic monitoring supports NHS guidelines on ethnic monitoring, ${ }^{26}$ "training of NHS and social care staff is critical to the success of collecting ethnic group data". On the basis of the findings presented here, we have developed an online training tool for telephonebased ethnic monitoring, which is provided as a supplement to this paper. This training tool is being rolled out by the cancer support and helpline service concerned, and the impact on calls will be assessed in a future evaluation.

This training tool is relevant to other telephone-based healthcare organisations. No such empirically based online tool has been developed before. The interactive training tool guides call-handlers through the different ways the ethnic monitoring question can be asked; and how problems such as caller uncertainty, resistance and call-handler presumption can be avoided, and the quality of the data gathered optimised. The tool provides participants with the opportunity for reflection on personal experience of asking the ethnicity monitoring question and discussion of the real examples used. It delivers specific recommendations on how best to ask the ethnic monitoring question over the telephone. The training tool provides a novel way in which training in ethnic monitoring can be delivered across a broad range of telephone delivered healthcare services.

\section{Strengths}

Our findings are based on the direct observation and analysis of what actually happens in cancer helpline calls, and how call-handlers actually manage ethnic monitoring in practice (and not, therefore, on recall, which is often faulty or incomplete). Moreover, the recommendations that emerge about effective techniques are derived from actual practice, and are not theory driven.

\section{Limitations}

Analysis was based on only one cancer helpline, although our findings resonate with research on ethnic monitoring in a range of environments, which points to the likely conceptual transferability of findings. It is difficult on the basis of this study design to assess the extent to which the resulting ethnic data are compromised by the kinds of interactional difficulties identified here.

\section{CONCLUSIONS}

In light of our findings, we recommend that telephonebased healthcare personnel who are responsible for ethnicity monitoring try to avoid asking the question in an 'open' format such as "Can I ask your ethnicity?" or "What is your ethnicity?" Instead, a question containing a (short) standardised list can assist callers in providing their response; future research might usefully explore the optimum number of ethnic categories that would be most appropriate in asking callers about their ethnicity. Given the possible sensitivity of the question, a preaccount explaining why an organisation is collecting the information can help to minimise the chance of causing offence, minimise caller resistance, and enhance call progressivity. Call-handler presumption can compromise the data collected. When a response is unclear, callhandlers are advised to check the accuracy of the ethnic category solicited before it is documented.

Acknowledgements National Institute of Health Research for funding this work via a personal Fellowship Award for GML. University of Southampton, Faculty of Medicine, for part-funding KE and GML. Dimbleby Cancer Care for funding-related work (including interviews with callers and call-takers and a survey of cancer helplines across the UK). The Helpline for their collaboration and open-mindedness. Callers to the helpline. Data workshop participants.

Contributors GML, KE and PD helped plan, design and coordinate the study. All authors provided input into the data analysis. The initial draft of the manuscript was prepared by GML and KE and then circulated repeatedly among all authors for critical revision. All authors read and approved the final manuscript.

Funding Support by the National Institute of Health Research (grant number 508332102) and Dimbleby Cancer Care (grant number 5079231010).

\section{Competing interests None.}

Ethics approval Faculty of Medicine Research Ethics Committee, University of Southampton.

Provenance and peer review Not commissioned; externally peer reviewed.

Data sharing statement Ethics approval does not permit the sharing of our audio-recorded helpline calls.

\section{REFERENCES}

1. Great Britain. Equality act 2010. Chapter 15. London: Stationery Office, 2010.

2. Mathur R, Grundy E, Smeeth L. Availability and use of UK based ethnicity data for health research. National Centre for Research Methods Working Paper, 2013:4-5. http://eprints.ncrm.ac.uk/3040/ (accessed 28 Mar 2013).

3. Raleigh V. Collection of data on ethnic origin in England. BMJ 2008;337:645-6.

4. Hull SA, Mathur R, Badrick E, et al. Recording ethnicity in primary care: assessing the methods and impact. Br J Gen Pract 2011;61:290-4.

5. Jack RH, Linklater KM, Hofman D, et al. BMC Public Health 2006;6:281.

6. Lawrenson R, Leydon G, Freeman G, et al. Are we providing for ethnic diversity in accident and emergency (A \& E) departments? Ethn Health 1998;3:117-23.

7. Pringle M, Rothera I. Ethnic group data collection in primary care: problems and solutions. Nottingham: University of Nottingham Medical School, 1995.

8. Kljakovic M. Is it easy collecting ethnicity data in general practice? N Z Med J 1993;106:103-4.

9. Fulton R. Ethnic monitoring: is health equality possible without it? Better health briefing 21. London: Race Equality Foundation, 2010.

10. National statistics. Ethnic group statistics: a guide for the collection and classification of ethnicity data. Norwich: HMSO, 2003.

11. Aspinall P. Operationalising the collection of ethnicity data in studies of the sociology of health and illness. Sociol Health IIIn 2001;23:829-62.

12. Bhopal RS. Ethnicity, race and health in multicultural societies: foundations for better epidemiology, public health and health care. Oxford: Oxford University Press, 2007.

13. Aspinall PJ, Jacobson B. Why poor quality of ethnicity data should not preclude its use for identifying disparities in health and healthcare. Qual Saf Health Care 2007;16:176-80. p. 176.

14. Wilkinson S. Constructing ethnicity statistics in talk-in-interaction: producing the 'White European'. Discourse Soc 2011;22:343-61.

15. Wilkinson S. Improving ethnic monitoring on a telephone helpline. In Antaki C, ed. Applied conversation analysis: intervention and change in institutional talk. Basingstoke: Palgrave Macmillan, 2011:75-97.

16. Iqbal G, Gumber A, Johnson M, et al. Improving ethnicity data collection or health statistics in the UK. Divers Health Care 2009;6:267-85. 
17. Leydon G, Ekberg K, Drew P. 'How can I help?' Nurse call openings on a cancer helpline and implications for call progressivity. Patient Educ Couns 2013;1:23-30.

18. Jefferson G. Glossary of transcript symbols with an introduction. In: Lerner G, ed. Conversation analysis: studies from the first generation. Philadelphia: John Benjamins, 2004:13-23.

19. Drew P, Chatwin J, Collins S. Conversation analysis: a method for research into interactions between patients and health-care professionals. Health Expect 2001;4:58-70.

20. Drew P. Conversation analysis. In: Fitch K, Sanders R, eds. Handbook of language and social interaction. London: Lawrence Erlbaum, 2004:71-102.

21. Sidnell J. Conversation analysis: an introduction. Cambridge: Cambridge University Press, 2010.

22. Baker C, Emmison M, Firth A. Calling for help: language and social interaction in telephone helplines. Amsterdam, Philadelphia: John Benjamins, 2005.
23. Heritage J, Maynard DW. Communication in medical care: interaction between primary care. Cambridge: Cambridge University Press, 2006

24. Schegloff $E$. Turn organization: one intersection of grammar and interaction. In: Ochs E, Schegloff E, Thompson SA, eds. Interaction and grammar. Cambridge: Cambridge University Press, 1996:52-133.

25. Aspinall P. Answer formats in British census and survey ethnicity questions: does open response better capture 'superdivirsity'? Sociology 2012;46:354-64.

26. Department of Health. A practical guide to ethnic monitoring in the NHS and social care. 2005. http://www.dh.gov.uk/prod consum dh/ groups/dh_digitalassets/@dh/@en/documents/digitalasset/dh_ 4116843.pdf 